\title{
Da keuchgrinste sie süßsäuerlich Über kopulative Verb- und Adjektivkomposita
}

\author{
1. Definition der Determinativ- und Kopulativkomposita \\ 2. Verbkomposita \\ 3. Adjektivkomposita \\ 3.1. Farbkomposita \\ 3.2. Adjektive, die Personengruppen bezeichnen \\ 3.3. Sonstige Adjektivkomposita \\ 3.3.1. Grundsätzliches \\ 3.3.2. Bildungen mit Synonymen \\ 3.3.3. Bildungen mit Antonymen \\ 3.3.4. Komposita mit Adjektiven aus gleichen und verschiedenen Paradigmen \\ 4. Zusammenfassung der Ergebnisse \\ 5. Literatur \\ 5.1. Belegquellen \\ 5.2. Forschungsliteratur
}

In der Wortbildung unterteilt man die Komposita traditionell in Determinativkomposita einerseits und Kopulativkomposita andererseits, wobei die determinative Komposition bei der Bildung neuer Sprachzeichen eine größere Rolle spielt. Der Anteil der Zusammensetzungen, die in der bisherigen Forschung zu den Kopulativkomposita gerechnet wurden, ist jedoch keineswegs gering. Allein die der Adjektive machen nach Pümpel-Mader et al. (1992, S. 43) „ca. $1 / 4$ des Gesamtcorpus" der von ihnen ermittelten Adjektivkomposita aus. Die Kategorie Kopulativkompositum ist also kein vernachlässigbares Randphänomen der Wortbildung und verdient eine genauere Betrachtung und Definition.

Die Definitionskriterien für Kopulativkomposita wurden verschiedentlich zaghaft relativiert (vgl. dazu z. B. den Forschungsüberblick bei Neuß 1981, S. 36-39). Aber erst Breindl/Thurmair (1992) haben die in der Forschung unbeirrt postulierte "fundamentale Zweiteilung“ (ebd. S. 32) in Determinativund Kopulativkomposita entschieden und grundsätzlich in Frage gestellt. Sie haben Substantivkomposita untersucht und zeigen anhand der Analyse von Bildungen wie Fürstbischof und Hosenrock, Kinocafé und Kleiderschürze, Dichterkomponist und Ministerfreund, daß es „keine nachweisbaren Kriterien gibt, die die Existenz zweier eindeutig distinkter Kategorien Kopulativkomposita und Determinativkomposita innerhalb der N-N-Komposita rechtfertigen" (ebd. S. 60). Für sie sind solche Bildungen ausschließlich Komposita, die neben der immer möglichen determinativen Lesart auch eine kopulative Interpretation zulassen, wobei - wie u. a. ihre Befragungstests ergaben - in vielen Fällen „die kopulative Interpretation gerade nicht die naheliegendste ist, ja 
vielmehr offensichtlich kontraintuitiv“ (ebd. S. 52). Substantivische „Kopulativkomposita lassen sich damit problemlos unter die (ohnehin weite und heterogene) Klasse der Determinativkomposita subsumieren“ (ebd. S. 60).

Ich schließe mich den Überlegungen Breindls und Thurmairs in bezug auf die von ihnen analysierten Substantivkomposita an und greife ihre Anregung auf, den Begriff Kopulativkompositum auch im Hinblick auf andere Wortarten neu zu überdenken. Dabei erweist sich ihre Entscheidung, den Forschungsgegenstand Kopulativkompositum nach Wortarten getrennt zu betrachten (ebd. S. 33), als sinnvoll: Zwar liegen die relativ einfachen Verhältnisse bei den Verbkomposita ähnlich wie bei den meisten substantivischen Zusammensetzungen. Doch ein großer Teil der Adjektivkomposita zeigt ganz spezifische Besonderheiten, die m. E. eine eigene Kategorie Kopulativkompositum nicht nur rechtfertigen, sondern notwendig machen.

Im folgenden soll also die Unterscheidung zwischen Determinativ- und Kopulativkomposita auch für die verbalen und adjektivischen Wortbildungsprodukte des Deutschen überprüft werden. Einigen der verbalen und adjektivischen Kompositatypen entsprechen Substantivkomposita, die bei Breindl/ Thurmair (1992) nicht behandelt worden sind. In diesen Fällen weise ich auf Zusammenhänge hin und untersuche, ob die Resultate von Breindl/Thurmair (1992) auch auf diese Komposita übertragbar sind oder ob es unter ihnen nicht doch rein kopulative Substantivkomposita gibt.

\section{Definition der Determinativ- und Kopulativkomposita}

Determinativkomposita werden definiert als binäre Strukturen, deren erste subordinierte Konstituente die zweite übergeordnete Konstituente determiniert, d. h. näher bestimmt (Hutschachtel, tomatenrot, radfahren). Das Zweitglied dominiert semantisch. Es repräsentiert jeweils das gesamte Kompositum: Eine Hutschachtel ist eine Schachtel, tomatenrot ist rot. Daher sind die Konstituenten nicht ohne Bedeutungsveränderung umkehrbar: Eine Hutschachtel ist kein Schachtelhut. Schließlich können die Konstituenten - im Gegensatz zu denen der Kopulativkomposita - gleichen oder verschiedenen Wortarten angehören: Geschirrschrank, Kühlschrank, Unterschrank.

Kopulativkomposita - die in der Forschungsliteratur verwirrenderweise u. a. auch als „,appositive“, „appositionelle“ oder „Komposita mit appositionellem Verhältnis" (Grimm 1826, zit. nach Breindl/Thurmair 1992, S. 33, Anm. 2, kritisch dazu Neuß 1981, S. 32), als „Dvandva“ (kritisch dazu Breindl/Thurmair 1992, S. $38 \mathrm{f}$.), als „koordinierte“, „koordinative“ bzw. „Koordinativkomposita“ (Lang 1977, S. 258), als „konjunktive Komposita“ (Kanngiesser 1985, zit. nach Fleischer/Barz 1992, S. 46), als ,attributive Komposita“, als „Additiva“ bzw. ,additionelle“ bzw. „,additive Komposita“ (Pümpel-Mader et al. 1992), als „Anreih-Komposita“, „Reihenwörter“, „Zwillingsformen“ oder „Verbindungszusammensetzungen" bezeichnet werden - unter- 
scheiden sich von den Determinativkomposita dadurch, daß ihre Konstituenten gleichgeordnet sind, d. h. auf keiner der beiden liegt das semantische Hauptgewicht; den gelegentlich geäußerten Eindruck, „,bei manchen liege das Gewicht auf der ersten Konstituente“ (ebenfalls skeptisch referiert bei Breindl/ Thurmair 1992, S. 46), kann ich nicht nachvollziehen. Aus der Gleichordnung der Konstituenten hat man abgeleitet, daß sie grundsätzlich ohne wesentliche Bedeutungsveränderung umkehrbar seien (vgl. u. a. Fleischer/Barz 1992, S. 246): süß-sauer bedeute sauer-süß (Erben 1993, S. 59; zur Bindestrichgraphie vgl. z. B. Gallmann 1989 und Starke 1989). Die Gleichordnung der Konstituenten erlaubt Zusammensetzungen aus mehr als zwei unmittelbaren Konstituenten (grün-gelb-blau), d. h. es können auch nicht-binäre Strukturen vorkommen. Die Gleichordnung der Konstituenten setzt schließlich deren Zugehörigkeit zur gleichen Wortart voraus, außerdem nach Lang (1977, S. 259) deren Zugehörigkeit zu einer „gemeinsamen Einordnungsinstanz“ („GEI“), nach Neuß (1981, S. 42) deren Zugehörigkeit zu ,demselben Paradigma“, nach Erben (1993, S. 59) deren Zugehörigkeit zur gleichen „Bezeichnungsklasse“.

Betrachten wir auf dem Hintergrund dieser Definitionen zunächst die gut überschaubaren Verbbildungen.

\section{Verbkomposita}

Auszuschließen sind hier Bildungen mit infiniter Verbform als Erstglied, z. B. liegenbleiben, stehenbleiben, spazierengehen, flötengehen, kennenlernen. Diese Bildungen gehören zu den trennbaren Verben, d. h. sie lassen bei einigen syntaktischen Aktualisierungen Distanzstellung zu, z. B. Schneewittchen blieb einfach im Sarg liegen. Häufig werden sie daher überhaupt nicht zu den Komposita gezählt. Will man sie aber als Komposita gelten lassen, sind sie immer Determinativkomposita, weil sie diesen in allen wesentlichen Kriterien entsprechen: Sie sind prinzipiell binär; das Erstglied bestimmt das Zweitglied genauer; das Zweitglied repräsentiert das gesamte Wort; die Konstituenten können nicht ohne Bedeutungsveränderung vertauscht werden.

Nichttrennbar sind hingegen Verbkomposita mit einem Verbstamm als Erstglied, z. B. ziehschleifen und keuchgrinsen. Solche in der Forschungsliteratur als kopulative Komposita angeführten Verbbildungen begegnen offenbar nur in der Fachsprache (Herstellungstechnik) und in der „(expressiven) Dichtung" (Wellmann 1995, S. 437), sind also textsortenbeschränkt.

Die fachsprachlichen Komposita kommen meist nur als infinite oder als Nominalformen vor, unterliegen mithin zusätzlichen Verwendungsbeschränkungen (vgl. „,seltene Ausnahmen“ bei Reinhardt 1966, S. 191: Man fließpreßt volle oder hohle Formteile). In der Forschungsliteratur überwiegend ohne Kotext angegebene Beispiele sind: brennhärten, tauchhärten, deckpolieren, Flammpolieren, preßglanzpolieren, sprengnieten, schwingschleifen (Reinhardt 1966, S. 186), glimmnitrieren (ebd. S. 194, Anm. 45), Feinziehschleifen, Kaltpreßschweißen, Schrägeinstechschleifen (Schütze 1969, S. 424 f.). 
Beispiele für Kopulativkomposita in belletristischen Texten sind: Ein Droschkentaxameter rollrasselt langsam über die blinzend[!] beleuchtete Landstraße (Kerr, zit. nach Schneider 1963, S. 205); Dickes schlammiges Wasser sprudelwallte, brodelte über ihnen (Döblin, zit. nach ebd.); Festliche Stimmung schwatzlachte an Bord (Ponten, zit. nach ebd.); Bevor der Berliner aber tadelt oder lobtadelt (Tucholsky, zit. nach Erben 1993, S. 59); Ich streute zu allem Überfluß noch kroßgebackene Schweinespeckbrösel über den Rohkostsalat [...] und knirschkaute genüßlich (Lander 1995, S. 110). Bei Fleischer/Barz (1992, S. 295): grinskeuchen, stöhnschnappen, zuckschlingen, schnaufwittern. Hier bestehen - anders als bei den fachsprachlichen Verbbildungen - keine besonderen Verwendungsbeschränkungen.

In beiden Bereichen kommen solche Verbkomposita äußerst selten vor. Sie gelten als stilistisch auffällig und erschweren die Rezeption - „der Leser stutzt" (Schneider 1963, S. 206). Daher ist die Hörer- bzw. Leserakzeptanz bei potentiell möglichen Bildungen wie etwa Er schlichspähte ... statt belegtem Er schlich hin und spähte in Ställe, Stuben und Gaden (Schneider 1994, S. 11) vermutlich eher gering. Stilistisch noch fragwürdiger und zudem noch leseunfreundlicher wären - $\mathrm{m}$. W. in der Belletristik nicht belegte, aber leicht bildbare - Komposita aus mehr als zwei Verben, z. B. Schneewittchen stöhnschnappschlang den vergifteten Apfel (vgl. den fachsprachlichen Beleg bei Reinhardt 1966, S. 191: Stauch-Reck-Schmieden). Bei diesen vom Sprachsystem grundsätzlich zugelassenen Komposita aus drei oder noch mehr Konstituenten könnte nun angenommen werden, es handele sich um nicht-binäre Strukturen. Während es jedoch bei den Adjektiven - wie unten zu erläutern sein wird Strukturen gibt, die nur tertiär etc. aufgelöst werden können (rot-weiß-blau), sind die potentiell möglichen Verbkomposita mit mehr als zwei Bestandteilen m. E. problemlos in jeweils zwei unmittelbare Konstituenten zu segmentieren: Ein Verb wie stöhnschnappschlingen wäre zu analysieren als linksverzweigtes Kompositum mit dem Determinans stöhnschnappen (weiter zu segmentieren in stöhnen und schnappen) und dem Determinatum schlingen.

Entscheidend für unsere Fragestellung ist nun, daß alle diese Verbbildungen nicht nur kopulativ, sondern immer auch determinativ interpretiert werden können: grinskeuchen kann je nach Ko- und Kontext sowohl kopulativ paraphrasiert werden (,gleichzeitig grinsen und keuchen'), als auch determinativ (,grinsend keuchen', ,mit einem breiten Grinsen keuchen', ,keuchen, wobei gegrinst wird' u. ä.). Für die fachsprachlichen Komposita nehmen Fleischer/ Barz (1992, S. 295) sogar an, daß determinative Lesarten grundsätzlich näherliegen, „da das Erstglied in der Regel als modale Spezifizierung des Zweitgliedes verstanden wird und eine Vertauschung der Reihenfolge der UK [ $=$ unmittelbaren Konstituenten] mit einer Bedeutungsveränderung verbunden ist, vgl. spülbohren - ,spülend bohren' [...], aber nicht bohrspülen“. „Wenn es sich auch um den gleichzeitigen Ablauf von zwei Vorgängen handelt, so ist doch der eine dem anderen in starkem Maße untergeordnet" (Reinhardt 1966, S. 192). Folgt man seiner Analyse (ebd. S. 191), nach der ,von den mit Kontext vorlie- 
genden Beispielen [...] nicht eines die Ausdeutung als Kopulativkompositum“ zuläßt, so „kritisiert Reinhardt [...] zu Recht die westdeutsche Duden-Grammatik, die diesen Typ (ziehschleifen) als kopulative Zusammensetzung zweier Verben ansieht" (Schütze 1969, S. 422).

Unter der Prämisse, daß Komposita, die determinativ paraphrasiert werden können, grundsätzlich zu den Determinativkomposita gerechnet werden müssen, stelle ich fest: Verbkomposita mit Verbstamm als Erstglied sind Determinativkomposita, auch wenn neben den determinativen Lesarten eine kopulative möglich ist.

\section{Adjektivkomposita}

Im Vergleich zu den insgesamt eher seltenen V-V-Komposita sind kopulativkomposita-verdächtige Adjektivbildungen in allen Textbereichen deutlich frequenter. Die Leitfrage meines Beitrags nach der Berechtigung einer eigenen Kategorie Kopulativkompositum kann daher auf dem Hintergrund eines geradezu abundanten Belegmaterials beantwortet werden.

Als erstes fallen Komposita, die Farben, und solche, die Personengruppen bezeichnen, auf. In eine dritte Gruppe können alle übrigen Komposita mit Adjektivkonstituenten gefaßt werden. Weil diese drei Gruppen z. T. recht verschiedenen Kompositionsbedingungen unterliegen, sollen sie im folgenden getrennt untersucht werden.

\subsection{Farbkomposita}

Einig scheint sich die Forschung darüber zu sein, daß ein Teil der aus Adjektiven gebildeten Farbkomposita hier ausgegrenzt werden muß, z. B. grün-graue Augen (Th. Mann, COSMAS), ein malerisch vor grün-blauer Seelandschaft postierter Mann (Die Zeit 1987, COSMAS), vom Frost blaurote Lippen, außerdem gelbgrün, blauschwarz, blaulila, schwarzbraun, rotorange, rotbraun $\mathrm{u}$. ä. Solche Komposita bezeichnen „Mischfarben“ (Neuß 1981, S. 49) und sind nach einem „modifikativen“ Wortbildungsmuster gebildet (Pümpel-Mader et al. 1992, S. 76). Sie sind also Determinativkomposita. Das Erstglied modifiziert jeweils das semantisch dominante Zweitglied: grau-grün bezeichnet eine grüne, jedoch etwas ins Graue gehende Farbe; blauschwarzer Samt ist schwarzer Samt, dessen tiefes Schwarz von einer dunklen Blautönung herrührt etc. Wie bei einigen anderen Determinativkomposita auch ist hier neben den primären determinativen Lesarten zusätzlich eine kopulative Interpretation möglich: So geht z. B. Wellmann (1995, S. 517) von einer ,medialen“ Beziehung zwischen den Konstituenten aus und versteht Farbkomposita diesen Typs als Bildungen, mit denen „Zwischenstufen (,bis') signalisiert" werden sollen. Nach seiner Auslegung wären also grünblaue Augen Augen, deren Farbe von grün bis blau changiert und folglich sowohl grün als auch blau ist (ähnlich Ortner/Ortner 1984, S. 66). 
Eine ganze Reihe anderer Farbkomposita hingegen ist ausschließlich kopulativ zu interpretieren, z. B. rot-weiß-rote Ärmelstreifen (Die Welt 1964, COSMAS), die weiß-grüne Flagge Sachsens (Sachsenspiegel 1990, COSMAS), ein rot-weißer Schlagbaum, ein gelbweißes Schachbrett, lila-weiße Kühe, ein rotblau-rosanes Bonbonpapier, schwarz-weiß-braune Schnürsenkel etc.

Im Vergleich zu den oben angeführten Verben sind solche Bildungen vielseitiger verwendbar. Dies belegen u. a. die zahlreichen linksverzweigten $\mathrm{Zu}$ sammensetzungen mit kopulativen Farbkomposita, z. B. rotgrüngelbschreiend wie die Fotos, die die Westverwandtschaft letzten Sommer in Italien schoß (Loest 1981, S. 19), ein rotweißkariertes Federbett (Fuchs 1992, S. 128). Möglich sind hier auch Komposita mit „morphologisch gekürzter A-Konstituente“ (Pümpel-Mader et al. 1992, S. 64), z. B. spinat-resedafarben (Knef, zit. nach ebd.).

Für diese Farbkomposita gilt: Sie können mehr als zweigliedrig sein; die Konstituenten entstammen demselben Paradigma; die letzte Konstituente kann nicht das gesamte Kompositum repräsentieren, d. h. die Konstituenten sind gleichwertig.

Allerdings sind die Konstituenten solcher Kopulativkomposita nur zu einem geringen Teil ohne Bedeutungsveränderung vertauschbar: ein gelbweißes Schachbrett z. B. ist ein weißgelbes Schachbrett. Zum großen Teil sind sie nur bedingt vertauschbar, u. a. weil das Zweitglied die im Gesamteindruck dominierende Farbe bezeichnet: Japans rotweiße Flagge, ein grünweißes Kleid. Letzteres bezeichnet für gewöhnlich ein weißes Kleid, das ein grünes Muster hat, z. B. auf weißem Grund grün gestreift oder gepunktet ist. Prinzipiell nicht umstellbar sind schließlich alle "Wörter mit additiv/sequentieller Relation" (Pümpel-Mader et al. 1992, S. 38), weil hier die Wortfolge auf eine bestimmte Farbabfolge referiert: eine weiß-rot-grüne Fahne ist eben nicht dasselbe wie eine grün-rot-weiße Fahne und eine rot-gelb-grüne Ampel ist etwas anderes als eine gelb-grün-rote Ampel. Dazu sei hier nur angemerkt: Durch diese Tatsache wird das Kriterium ,Vertauschbarkeit der Konstituenten' als Definitionskriterium für Kopulativkomposita zweifelhaft. Ich komme unten (vgl. 3.2. und 3.3.) noch einmal ausführlicher darauf zurück.

Dem hier beschriebenen Adjektivtyp entsprechen Substantivbildungen wie das Weiß-Rote des Schlagbaums, das Weiß-Rot-Grüne der Flagge usw. Ich gehe davon aus, daß es sich hierbei um Derivate von kopulativen Adjektivkomposita handelt (weiß-rot $\rightarrow$ das Weiß-Rote) und nicht um Komposita aus Substantivderivaten (weiß $\rightarrow$ das Weiße, rot $\rightarrow$ das Rote), zumal es z. B. im letzten Fall eigentlich das Weiße-Rote heißen müßte. Dies vorausgesetzt, gibt es auch im Bereich der Farbsubstantive keine kopulativen Komposita.

Ich fasse zusammen: Für einen Teil der Farbkomposita muß eine eigene Kategorie Kopulativkompositum angesetzt werden.

\subsection{Adjektive, die Personengruppen bezeichnen}

Einen weiteren eigenen Typ von Adjektivkomposita bilden solche, die die $\mathrm{Zu}$ gehörigkeit zu einer bestimmten Personengruppe bezeichnen. Dies betrifft vor allem ethnische oder Gruppen verschiedener Staatsangehörigkeit, z. B. ameri- 
kanisch-israelische Geschäftsleute, deutsch-polnische Freundschaft, deutsch-dänisch-schwedische Verhandlungen.

Auch für diese Komposita gilt: Sie können mehr als zweigliedrig sein; die Konstituenten entstammen demselben Paradigma; die letzte Konstituente kann nicht das gesamte Kompositum vertreten, vielmehr treffen alle durch die Adjektive ausgedrückten Eigenschaften gleichermaßen zu.

Es lassen sich drei Haupttypen unterscheiden:

Zum einen wird ausgedrückt, daß ein Bezugswort, meist eine Person bzw. Personengruppe oder Institution, sowohl die Eigenschaft $A$ als auch die Eigenschaft B und eventuell noch weitere Eigenschaften C, D, E usw. besitzt, z. B. der deutsch-israelische Publizist Henryk M. Broder (Die Zeit 1986, COSMAS), ein deutsch-französisches Internat.

Einen zweiten Typ bilden Komposita, mit denen Beziehungen zwischen Personen oder Institutionen u. ä. ausgedrückt werden: deutsch-polnische Verhandlungen sind Verhandlungen zwischen deutschen und polnischen Verhandlungspartnern; die deutsch-polnische Grenze befindet sich zwischen dem deutschen und polnischen Staatsgebiet; vgl. auch ,linguistisch-sozialwissenschaftliche Zusammenarbeit $\rightarrow$,Z., (die) zwischen Vertretern der linguistischen und sozialwissenschaftlichen Disziplinen (besteht) “ (Pümpel-Mader et al. 1992, S. 36). In diesen Fällen sind die Bezugswörter ausschließlich solche, die bereits eine Interaktion zwischen den im Adjektivkompositum thematisierten Partnern ausdrücken: Verhandlungen, Gespräche, Zusammenarbeit, Vertrag, Abkommen, Warenverkehr, Austausch, Freundschaft, Feindschaft, Frieden, Krieg usw. Dieses Muster von Adjektivkomposita ist hochproduktiv, aus Sachgründen naheliegenderweise vor allem in politischen Essays, in Zeitungsartikeln oder Nachrichten- und Magazinsendungen.

Ähnliche semantische Besonderheiten wie dieser zweite Typ der Adjektivkomposita weisen die bei Breindl/Thurmair (1992) explizit ausgeklammerten Appellativa auf wie Baden-Württemberg, Österreich-Ungarn, die jeweils den Zusammenschluß mehrerer Elemente zu einem (neuen) Ganzen zum Ausdruck bringen: Österreich-Ungarn ist keineswegs, Ungarn, dem Österreich angegliedert ist', ,Ungarn mit Österreich' o. ä. Vielmehr beinhalten die Komposita das gleichwertige und parallele Vorhandensein beider Konstituentenbedeutungen, sind also Kopulativkomposita.

Zum dritten adjektivischen Typ gehören schließlich Komposita, die eine zeitliche Abfolge signalisieren, z. B. „ottonisch-salische Zeit $\rightarrow, Z$. der Ottonen und - danach - der Salier “ (Pümpel-Mader et al. 1992, S. 36).

Die Zusammensetzungen des ersten und zweiten Typs sind im Prinzip umstellbar, wenn auch bestimmte Reihenfolgen präferiert werden, u. a. solche, die durch semantische Gewichtungen vorgegeben werden (vgl. Sommerfeldt 1984, S. 96), bzw. bestimmte Reihenfolgen, die durch Namensbildungen festgelegt werden (z. B. in deutsch-sowjetische Freundschaft). Nicht umstellbar sind die Komposita des dritten Typs, bei denen die Reihenfolge der Konstituenten auf 
eine zeitliche Abfolge referiert. Damit erhärten sich meine bereits im Zusammenhang mit den kopulativen Farbadjektiven konstatierten Zweifel am Definitionskriterium ,Vertauschbarkeit der Konstituenten`.

Alle drei Adjektivtypen können nur kopulativ gelesen werden. Typisch determinative Paraphrasierungen sind - jedenfalls meiner Sprachkompetenz nach - ausgeschlossen: eine deutsch-sowjetische Freundschaft ist keine ,auf deutsche Art sowjetische Freundschaft', eine ottonisch-salische Zeit ist keine ,auf ottonische Weise salische Zeit ${ }^{\text {. }}$.

Ich fasse zusammen: Für Adjektivkomposita, die auf die Zugehörigkeit zu ethnischen oder Gruppen verschiedener Staatsangehörigkeit referieren, und für solche, die Beziehungen zwischen Personen, Personengruppen, Institutionen etc. bezeichnen, sowie für Adjektivkomposita, die eine zeitliche Aufeinanderfolge verschiedener Elemente ausdrücken, ist eine eigene Kategorie Kopulativkompositum anzusetzen.

\subsection{Sonstige Adjektivkomposita}

\subsubsection{Grundsätzliches}

Bei den sonstigen Komposita mit adjektivischen Konstituenten sind wiederum zunächst auszuschließen: Determinativkomposita, die nur determinative Lesarten haben, und Determinativkomposita, die neben den determinativen auch eine kopulative Lesart erlauben, z. B. „verrückt-diktatorischer König (Hörbeleg) $\rightarrow$,K., der verrückt und diktatorisch ist" “ neben „modifikativem“, also determinativem, „,K., der in verrückter Art und Weise diktatorisch ist" “ (Pümpel-Mader et al. 1992, S. 41). Nach ebendiesem Interpretationsverfahren lassen sich weitere - z. B. ebd. (S. 39) oder bei Wellmann (1995, S. 515) - als kopulativ aufgefaßte Komposita analysieren: Für höflich-bestimmt oder friedlich-leeres Gesicht usw. liegen m. E. auch determinative Paraphrasen nahe wie ,auf höfliche Art bestimmt (auftretend)', ,ein auf friedliche Art leer aussehendes Gesicht'. Hierzu rechne ich aus meiner eigenen Belegsammlung: stumpfsinnig-tückisch (Fuchs 1992, S. 16), mit einem triumphierend-verzweifelten Lächeln (ebd. S. 31), Friedlich-freundlich saßen sie zusammen (Loest 1981, S. 299). Auch Sommerfeldt (1984, S. 95) weist darauf hin, daß Komposita wie raukkalt, naßkalt, feuchtwarm ,an der Peripherie der kopulativen Komposita“ stehen, „da die erste Konstituente auch als Präzisierung der zweiten aufgefaßt werden kann“. Als Präzisierung gilt bei ihm „, kalt und noch dazu naß“ “ (ebd.); hier bieten sich weitere Paraphrasierungen an, die noch deutlicher auf Determination hinweisen, z. B. ,kalt und zwar auf nasse Art', ,auf nasse Art kalt', ,auf feuchte (und nicht auf die übliche trockene) Weise warm' etc.

Auch viele Komposita mit mehr als zwei Bestandteilen, z. B. fruchtigsahnig-süßer Duft (Mini-Amica S. 11, Beilage zu Amica 1996, Heft 3) können als binäre Strukturen segmentiert und determinativ interpretiert werden, z. B. ,ein in fruchtig-sahniger Weise süßer Duft'. 


\subsubsection{Bildungen mit Synonymen}

Ebenfalls als Determinativkomposita einzuordnen sind m. E. die bei PümpelMader et al. (1992, S. 53) „verdeutlichende Gleichsetzungskomposita“ genannten Adjektivbildungen, z. B. nichtssagend-stumpf (Seghers, zit. nach Neuß 1981, S. 58). Pümpel-Mader et al. (1992, S. 55-61) unterscheiden bei diesem „äquativ-explikativen“ Typ den „präzisierenden“, z. B. aromatisch-bitter, rundeiförmig, den „generalisierenden“, z. B. physisch-stofflich, das Gedanklich-Abstrakte, und den „gleichsetzenden“ Untertyp, z. B. alltäglich-gewöhnlich, süßniedlich, absichtsvoll-willentlich. Mitunter kommen auch verdeutlichende Bildungen mit Lehnwort und indigenem Bestandteil vor, z. B. einfach-primitiv, körnig-kristallin, privat-vertraulich (ebd. S. 62-64), eitelnarzißtisch (Mannheimer Morgen 1987, COSMAS).

„Als Paraphrasenkonstante [...] steht ,bzw. [...], ,nämlich' [...], ,genauer gesagt “ “ (Pümpel-Mader et al. 1992, S. 53), „, anders gesagt “ “ (Ortner/Ortner 1984, S. 149) oder „,d. h.“ “ (Helbig/Buscha, zit. nach Pümpel-Mader et al. 1992, S. 54). Pümpel-Mader et al. (1992, S. 53 ff.) sehen hier eine Gleichordnung der Konstituenten gegeben; zudem werden die genannten Paraphrasen häufig als Indiz für Kopulativkomposita angeführt. Dem kann ich mich nicht anschließen. Vielmehr meine ich, daß hier determinative Subordination vorliegt. Dies impliziert ja auch bereits der von ihnen selbst formulierte Terminusbestandteil „verdeutlichend“ bzw. „explikativ“: Bei den genannten Bildungen grenzt das Erstglied das Zweitglied durch „Verdeutlichung“ bzw. „Explikation" semantisch ein, indem es nämlich Aspekte des Zweitgliedes auf eine bestimmte Weise präzisiert, erweitert oder ergänzt. Dies funktioniert, weil es bekanntlich zwischen verschiedenen Sprachzeichen keine echte Synonymie gibt, weil ein Sprachzeichen einem anderen ähnlichen gegenüber immer noch zusätzliche Aspekte besitzt. Auch diese Zusammensetzungen gehören folglich zu den Determinativkomposita, die eine kopulative Lesart nicht ausschließen.

Ähnlich verhält es sich mit den bei Fleischer/Barz (1992, S. 247) „tautologische Kopulativkomposita“ genannten Bildungen, ,wo die beiden UK [ = unmittelbaren Konstituenten] synonymisch gefaßt werden könnten“, z. B. zierlich-zarte Frau (Goethe, zit. nach Fleischer/Barz 1992, S. 247). Auch hier liegen m. E. nicht Kopulativ-, sondern Determinativkomposita vor: Es handelt sich nämlich keineswegs um Synonyme, denn z. B. das die Gestalt betreffende zierlich bedeutet nicht dasselbe wie das die physische oder auch psychische Konstitution betreffende zart. Die Konstituenten sind folglich nicht gleichgeordnet, vielmehr modifiziert auch hier das Erst- das Zweitglied. Die ebd. angeführten Bildungen mit gegenwartssprachlich nicht mehr motivierten Konstituenten können in unserem Zusammenhang außer acht gelassen werden.

Allenthalben als Determinativkomposita definiert werden die bei Fleischer/Barz (1992, S. 247) „expressive Doppelungen“ genannten, im wesentlichen auf belletristische Texte beschränkten Adjektivbildungen aus zwei auch ausdrucksseitig - identischen Adjektiven, z. B. mit tief-tiefer Sammet- 
bläue (Th. Mann, zit. nach Fleischer/Barz 1992, S. 232), grau-graue Hemden (Strittmatter, zit. nach ebd.), billig-billig in Portugal drehen (Spiegel, zit. nach Pümpel-Mader et al. 1992, S. 79), Aber es kann gar nicht anders sein, als daß alles brav-brav gewesen ist (Loest 1981, S. 305). Hier determiniert das Erstglied das Zweitglied insofern, als es zu dessen Elation dient, wie bei ultratief, kreuzgrau, superbillig, stockbrav. Paraphrasiert werden kann z. B. mit ,besonders tief", ,besonders grau' etc.

Ich fasse zusammen: Adjektivkomposita mit mehr oder weniger synonymen oder mit identischen Konstituenten sind Determinativkomposita, die u. U. eine kopulative Lesart zulassen.

\subsubsection{Bildungen mit Antonymen}

Meist generell als Kopulativbildungen interpretiert werden die „Oxymora“ genannten Komposita aus antonymen Konstituenten, die ebenfalls vorrangig in belletristischen Texten vorkommen, z. B. Die [...] Kreiselrichtung des Pendels kann gut-böse, gefährlich-harmlos, heilsam-schädlich oder derlei bedeuten (Haefs 1993, S. 111), ein gebieterisch-schüchterner Schulmeister (Broch 1950, S. 163), mit der behutsam-unerbittlichen Sonde des Arztes (Th. Mann, COSMAS), finster-fröhlich dem Untergang entgegen (Die Zeit 1987, COSMAS), heißkühl (Broch, zit. nach Neuß 1981, S. 47), ernstheiter (Goethe, zit. nach Fleischer/Barz 1992, S. 247), außerdem bittersüß, süßsauer usw. Eine Sondergruppe bilden Oxymora mit „,morpholektisch" antonymen Konstituenten“ (Pümpel-Mader et al. 1992, S. 40), z. B. gezügelt-ungezügelte Leidenschaft, politisch-apolitische Radikalität (ebd.), in weiterem Sinne wohl auch: unheilvollheilsames Getränk (Durbridge, zit. nach Ortner/Ortner 1984, S. 171), Die Stimme hatte nichts von ihrer gewohnten interessiert-interesselosen Trockenheit verloren (Broch 1950, S. 217).

Antonymische Komposita fallen durch folgende Besonderheiten aus der obigen Definition von Kopulativkomposita heraus: Oxymora können m. E. prinzipiell nicht mehr als zwei unmittelbare Konstituenten haben, weil das Antonymie-Modell eine binäre Struktur voraussetzt; jedem Sprachzeichen am einen Ende einer gedachten Eigenschaftsskala steht nämlich jeweils ein semantisch konträres Sprachzeichen am entgegengesetzten Ende dieser Skala gegenüber. Außerdem wird die ,additiv/adversative Beziehung“ von Oxymora nicht durch ,und' u. ä., sondern in „Paraphrasenvarianten mit ,aber', genauer mit ,aber auch“ und ,aber trotzdem '/,dennoch\%,dafür' “ (Pümpel-Mader et al. 1992, S. 39) wiedergegeben.

Erstaunlich ist, daß einerseits die Konstituenten der meist pauschal als Kopulativkomposita ausgegebenen Oxymora zwar der Definition gemäß grundsätzlich umstellbar sein sollen, z. B. süß-sauer/sauer-süß (Erben 1993, S. 59), daß aber andererseits die Gegenüberstellung von Oxymora mit jeweils vertauschter Konstituentenreihenfolge in manchen Ko- und Kontexten gerade als zwingendes Argument gegen deren Einordnung in die Kopulativkomposita 
angeführt werden kann. So beobachten Fleischer/Barz (1992, S. 247), daß es sich bei Oxymora nicht immer um Kopulativkomposita handeln muß; „so spielt der Autor mit einem determinativen Verhältnis antonymischer UK [= unmittelbarer Konstituenten] in folgendem Satz: Er kann sowohl scharfgütig als auch gütig-scharf sein (Strittmatter)". Hier wird offensichtlich jeweils die Eigenschaft akzentuiert, die das Zweitglied bezeichnet. Die Konsequenzen aus dieser Beobachtung sind $\mathrm{m}$. E., daß jedes Oxymoron daraufhin untersucht werden muß, ob das semantische Hauptgewicht im jeweiligen Ko- und Kontext auf der zweiten Konstituente liegt und daher determinative Paraphrasierungen möglich macht, wie z. B. ,gütig, aber auf scharf(züngig)e Art', ,sauer mit einer süßen Note' etc. In den Fällen, in denen dies zutrifft, liegen Determinativkomposita vor.

Andererseits können solche Oppositionen in manchen Ko- und Kontexten gerade ein Indiz für Kopulativkomposition sein, z. B. Also dran denken: softknackig und nicht knackig-soft (TV-Werbung für Daim-Schoko-Riegel, ARD 18. 4. 1996). In diesem Hörbeleg wird ein Schoko-Riegel, der außen einen soften Überzug und innen einen knackigen Krokantkern hat, also beim Hineinbeißen erst soft und dann knackig ist, mit einem - den Aufmerksamkeitswert der Werbebotschaft erhöhenden - Gürteltier verglichen, das außen knakkig und innen soft sei. Es treffen also die in den Konstituenten ausgedrückten konträren Eigenschaften gleichermaßen und zwar in genau der genannten räumlichen bzw. zeitlichen Abfolge zu; determinative Lesarten sind ausgeschlossen.

Komposita, mit denen auf die Abfolge gegensätzlicher Eigenschaften referiert wird, heißen bei Pümpel-Mader et al. (1992, S. 39) ,,adversativ/sequentiell". Die diesen Bildungstyp illustrierenden Belege, z. B. schnell-langsame Musik (ebd.), zeigen - im Gegensatz zu dem vorigen Beleg - Komposita, deren Konstituenten ohne Bedeutungsveränderung vertauschbar sind, weil hier keine feste Reihenfolge, sondern der zumindest einmalige, in der Regel aber wiederholte Wechsel beider Eigenschaften zum Ausdruck kommen soll. Vgl. auch heiß-kalte Wechselduschen zum Abhärten (Mannheimer Morgen 1986, COSMAS).

Solche verwirrenden, weil z. T. widersprüchlichen Beobachtungen zur Vertauschbarkeit der Konstituenten, die schon oben bei der Analyse anderer Adjektive gemacht wurden (vgl. 3.1. und 3.2.), haben auch Breindl/Thurmair (1992, S. 46) zu dem konsequenten Schluß veranlaßt, „daß die Vertauschbarkeitsthese, die oft sogar als Definiens von Kopulativkomposita zitiert wird, in dieser kategorischen Form nicht haltbar ist" (vgl. in diesem Sinne ebenfalls einschränkend Günther 1993, S. 340). Dies kann ich nun zusätzlich im Hinblick auf die Adjektivkomposita bestätigen: Die Konstituenten einiger adjektivischer Kopulativkomposita sind ebenso wie die Konstituenten aller Determinativkomposita nicht vertauschbar. Eine Abgrenzung beider Kategorien auf der Basis des Kriteriums, Vertauschbarkeit' wäre folglich nicht plausibel. 
Ich fasse zusammen: Keineswegs alle Oxymora sind Kopulativkomposita. Das Kriterium ,Vertauschbarkeit der Konstituenten ohne wesentliche Bedeutungsveränderung' ist nicht geeignet, Kopulativkomposita sinnvoll zu definieren.

\subsubsection{Komposita mit Adjektiven aus gleichen und verschiedenen Paradigmen}

Ebenso wie das zur Definition der Kopulativkomposita immer wieder herangezogene Kriterium ,Vertauschbarkeit der Konstituenten' ist auch das Kriterium, gleiches Paradigma' fragwürdig. Dies soll im folgenden erläutert werden.

Wie oben (vgl. 1.) gesagt wurde, setzt die Gleichordnung der Konstituenten deren Zugehörigkeit zu der gleichen Wortart voraus. Darüber hinaus sollen die Konstituenten nach Lang (1977, S. 259) auch zu einer „gemeinsamen Einordnungsinstanz“" gehören, nach Neuß (1981, S. 42) zu „demselben lexikalischen Paradigma“, nach Erben (1993, S. 59) zur gleichen „Bezeichnungsklasse".

Auf Adjektivkomposita wie taubstumm trifft dies zu. Lang (1977, S. 259) nennt hier als „gemeinsames Fundierungsmerkmal“ die „organische Unfähigkeit“ mit den „Spezifizierungshierarchien: decodieren/encodieren“. Für Zusammensetzungen wie kleindicklich und breitflach könnte als gemeinsames Merkmal ,Gestalt" oder ,Form' angegeben werden, vgl. Diese kleindickliche, dauergewellte Frau mit etwas breitflacher Nase (Lander 1995, S. 81). Auch für die oben diskutierten antonymen Zusammensetzungen lassen sich gemeinsame Merkmale finden: „sü $\beta$, sauer und bitter gehören zum Paradigma der adjektivisch gefaßten Geschmacksqualitäten. [...] J. Erben zielt ebenfalls auf diesen Sachverhalt, wenn er als Bedingung für Kopulativkomposita fordert, daß sie der gleichen ,Bezeichnungsklasse' angehören müssen" (Neuß 1981, S. 43).

Auf Adjektive wie in ein großes, mager-strenges Mädchen (Fuchs 1992, S. 19) trifft dies allerdings nicht zu. Im Gegenteil: Hier werden Eigenschaften ganz verschiedener Bereiche aktualisiert. Zahlreiche - vor allem belletristische - Belege zu diesem Typ finden sich bei Pümpel-Mader et al. (1992, S. 45 f.), z. B. grobroter Kokosläufer und gelbsüße Baumwurst (beide Strittmatter), süßbraun (Benn), spitzgelbe Zähne und spitzbunte Tüten (beide Grass), blausüß (Mürr), schmalsilberne Mondsichel (Zuckmayer); vgl. außerdem rosanasse Nase (Ortner/Ortner 1984, S. 149), die Leere des ihm wohlbekannten steinern-dreiekkigen Platzes (Broch 1950, S. 198). Es fällt sofort auf, daß diese Zusammensetzungen nur kopulativ interpretiert werden können - und zwar gerade weil sie verschiedenen Paradigmen entstammen: ,Farbe' kann keinesfalls durch ,Geschmack' oder ,Form' determiniert werden, also z. B. gelb weder durch süß noch durch spitz. Derart unterschiedliche Eigenschaften können sich nur in den Fällen determinieren, in denen Metaphorisierungen vorliegen, z. B. da stach [...] weiß-scharf das Schweißlicht hervor (Broch 1950, S. 162).

Es gibt also einerseits eindeutig kopulative Komposita, deren Konstituenten über keine gemeinsamen Merkmale verfügen; andererseits gibt es determi- 
native Komposita, deren Konstituenten gemeinsame Merkmale haben, so u. a. Mischfarbenkomposita wie rotbraun, rotorange etc. (vgl. oben 3.1.) oder soziale Eigenschaften bezeichnende Komposita wie herablassend-freundlich (Hildesheimer, zit. nach Fleischer/Barz 1992, S. 245), kalt-egoistisch (Die Zeit 1985, COSMAS). Kriterien wie „gemeinsame Fundierungsmerkmale“ (Lang 1977, S. 259) helfen demnach bei der Differenzierung von Komposita nicht weiter.

Übrigens ist nach Eisenberg (1994, S. $321 \mathrm{ff}$.) auch für syntaktische Koordinationsstrukturen mit kopulativen Konjunkten, für die Lang (1977) primär die Erklärungsthese der „GEI“ vorgeschlagen hat, die Annahme gemeinsamer Merkmale keineswegs zwingend.

Ich fasse zusammen: Das Kriterium ,gemeinsame Einordnungsinstanz („GEI“), ,gleiches Paradigma“ oder ,gleiche Bezeichnungsklasse“ eignet sich nicht zur Abgrenzung der Kopulativ- von den Determinativkomposita.

\section{Zusammenfassung der Ergebnisse}

Meine Analyse von Verb- und Adjektivkomposita, die in der Forschungsliteratur immer wieder als Kopulativkomposita definiert werden, hat ergeben:

Es gibt keine kopulativen Verbkomposita.

Es gibt kopulative Adjektivkomposita im Bereich der Farbadjektive, der Adjektive, die Personengruppen bezeichnen, der Adjektive mit Antonymen und schließlich der Adjektive, die Eigenschaften aus gleichen oder verschiedenen Paradigmen komponieren.

Die häufig angeführten Definitionskriterien ,Vertauschbarkeit der Konstituenten' und ,gleiches Paradigma' eignen sich nicht zur Definition von Kopulativkomposita. Es gilt das Minimalkriterium, gleiche Wortart'. Ausschlaggebend ist das Kriterium ,Gleichordnung der Konstituenten', das sich in bestimmten Paraphrasen zeigt.

\section{Literatur}

\subsection{Belegquellen}

Aus der Forschungsliteratur entnommene Belege sind mit entsprechenden Zitatangaben versehen. Die mit COSMAS markierten Belege habe ich in den Korpora des Instituts für deutsche Sprache, Mannheim, recherchiert.

Eigene in diesem Beitrag zitierte Quellen sind:

Amica 1996. [Frauenmagazin]

Broch, Hermann (1950/1974): Die Schuldlosen. Roman in elf Erzählungen. Frankfurt: Suhrkamp (= Kommentierte Werkausgabe Bd. 5).

Fuchs, Gerhard (1992/1995): Katharinas Nacht. Roman. München: dtv.

Haefs, Gisbert (1993): Matzbachs Nabel. Kriminalroman. München: Goldmann. 
Lander, Jeanette (1995): Überbleibsel. Eine kleine Erotik der Küche. Berlin: Aufbau. Loest, Erich (1981/1992): Durch die Erde ein Riß. Ein Lebenslauf. München: dtv. Schneider, Robert (1992/1994): Schlafes Bruder. Leipzig: Reclam.

\subsection{Forschungsliteratur}

Breindl, Eva/Thurmaier, Maria (1992): Der Fürstbischof im Hosenrock. Eine Studie zu den nominalen Kopulativkomposita des Deutschen. in: Deutsche Sprache 20, S. $32-61$.

Eisenberg, Peter (1994): Grundriß der deutschen Grammatik. 3., überarbeitete Auflage. Stuttgart/Weimar: Metzler.

Erben, Johannes (1993): Einführung in die deutsche Wortbildungslehre. 3., neubearbeitete Auflage. Berlin: Erich Schmidt (= Grundlagen der Germanistik 17).

Fleischer, Wolfgang/Barz, Irmhild/unter Mitarbeit von Marianne Schröder (1992): Wortbildung der deutschen Gegenwartssprache. Tübingen: Niemeyer.

Gallmann, Peter (1989): Syngrapheme an und in Wortformen. Bindestrich und Apostroph im Deutschen. In: Eisenberg, Peter/Günther, Hartmut (Hgg.): Schriftsysteme und Orthographie. Tübingen: Niemeyer, S. 85-110.

Günther, Hartmut (1993): Kopulativkomposita. In: Glück, Helmut (Hg.): Metzler Lexikon Sprache. Stuttgart/Weimar: Metzler, S. 340.

Lang, Ewald (1977): Semantik der koordinativen Verknüpfung. Berlin: Akademie-Verlag (= studia grammatica XIV).

Neuß, Elmar (1981): Kopulativkomposita. In: Sprachwiss. 6, S. 31-68.

Ortner, Hanspeter/Ortner, Lorelies (1984): Zur Theorie und Praxis der Kompositaforschung. Mit einer ausführlichen Bibliographie. Tübingen: Narr (= Forschungsberichte des Instituts für deutsche Sprache Mannheim 55).

Pümpel-Mader, Maria/Gassner-Koch, Elsbeth/Wellmann, Hans/unter Mitarbeit von Lorelies Ortner (1992): Deutsche Wortbildung. Typen und Tendenzen in der Gegenwartssprache. Eine Bestandsaufnahme des Instituts für deutsche Sprache, Forschungsstelle Innsbruck. Fünfter Hauptteil: Adjektivkomposita und Partizipialbildungen (Komposita und kompositionsähnliche Strukturen 2). Berlin/New York: de Gruyter (= Sprache der Gegenwart LXXX).

Reinhardt, Werner (1966): Produktive verbale Wortbildungstypen in der Fachsprache der Technik. In: Wiss. Zs. der Päd. Hochschule Potsdam 10, S. 183-195.

Schneider, Wilhelm (1963): Stilistische deutsche Grammatik. Die Stilwerte der Wortarten, der Wortstellung und des Satzes. Dritte Auflage. Freiburg/Basel/Wien: Herder.

Schütze, Ruth (1969): „Außenrund-Schnelleinstechschleifen“. Bemerkungen zu einem Wortbildungstyp in der Fachsprache der Technik. In: PBB (Halle) 6, S. 421-426.

Sommerfeldt, Karl-Ernst (1984): Zur Reihenfolge der Konstituenten kopulativer Komposita. In: Sprachpflege 33, S. 93-96.

Starke, Günter (1989): Vom Sinn und Unsinn der Bindestrichschreibung. In: Deutschunterricht 42, S. 186-192.

Wellmann, Hans (1995): Die Wortbildung. In: Duden. Grammatik der deutschen Gegenwartssprache. 5., völlig neu bearbeitete und erweiterte Auflage. Herausgegeben und bearbeitet von Günther Drosdowski in Zusammenarbeit mit Peter Eisenberg, Hermann Gelhaus, Helmut Henne, Horst Sitta und Hans Wellmann. Mannheim/ Leipzig/Wien/Zürich: Dudenverlag, S. 399-539 (= Der Duden in 12 Bänden 4).

Adresse der Verfasserin:

Dr. Elke Donalies, Institut für Deutsche Sprache, R 5, 6-13, 68161 Mannheim 